\title{
Radiation recall pneumonitis in the setting of immunotherapy and radiation: a focused review
}

\section{Future Science} $\mathrm{OA}$

\author{
Kerri McGovern'1, Maged Ghaly², Michael Esposito³ ${ }^{3}$ Keara Barnaby \& Nagashree \\ Seetharamu*,1 \\ ${ }^{1}$ Division of Hematology \& Medical Oncology, Donald \& Barbara Zucker School of Medicine at Hofstra/Northwell, Lake Success, \\ NY, 11042 USA \\ ${ }^{2}$ Department of Radiation Oncology, Donald \& Barbara Zucker School of Medicine at Hofstra/Northwell, Lake Success, NY, 11042 \\ USA \\ ${ }^{3}$ Department of Pathology, Donald \& Barbara Zucker School of Medicine at Hofstra/Northwell, Lake Success, NY, 11042 USA \\ *Author for correspondence: nseetharamu@northwell.edu
}

Radiation recall pneumonitis (RRP) is an entity described as pneumonitis localized to a previously irradiated field after exposure to a systemic agent. It has previously been described in the literature in the context of chemotherapeutic agents as well as certain biologics. With immunotherapy taking a more prominent role in the treatment of several different malignancies and its own baseline risk of pneumonitis, it is important to explore the likelihood of RRP, specifically in those patients who have been previously treated with radiation therapy. The current literature regarding RRP with checkpoint inhibitors is reviewed in this article. Alongside this review, we report a case of RRP after pembrolizumab initiation in a patient in our practice.

Lay abstract: When patients undergo radiation to the chest there is a risk of inflammation to the lung tissue called pneumonitis. This inflammation can also be a result of certain medications, including checkpoint inhibitors, which are used to treat cancers. There is a specific phenomenon, radiation recall pneumonitis, where patients who receive radiation to the chest followed by checkpoint inhibitors experience pneumonitis only in the lung fields that were exposed to previous radiation. This paper reviews the existing body of literature and describes a case of radiation recall pneumonitis seen in our practice.

First draft submitted: 9 November 2018; Accepted for publication: 1 February 2019; Published online: 10 April 2019

Keywords: checkpoint inhibitors $\bullet$ immunotherapy $\bullet$ pneumonitis $\bullet$ radiation recall pneumonitis $\bullet$ thoracic radiation

Radiation recall is an unpredictable, poorly understood entity wherein an acute inflammatory response confined to a previously irradiated area ensues upon exposure to a systemic agent. Most of the cases of radiation recall reported in the literature are cutaneous, but a handful of reports pertaining to other sites have been reported. In this report, we focus on radiation recall pneumonitis (RRP), which is considered a type of radiation-related lung injury that presents as acute inflammation limited to previously irradiated lung fields, weeks, months or even years after completion of radiation therapy in response to a systemic trigger. Many systemic agents have been reported to trigger RRP but to our knowledge, there is very limited literature on RRP induced by checkpoint inhibitors. In this manuscript, we report a case of PD-1 inhibitor-associated RRP and present a focused review on the topic.

\section{Etiopathogenesis of RRP}

The mechanistic details of RRP are not completely characterized. However, several mechanisms have been postulated [1]; the foremost of them is that the stem cells in irradiated tissue are subject to sublethal damage resulting in aberrant function including their ability to proliferate. Subsequent generations of cells in that same irradiated tissue become more sensitive and mount an inflammatory response in the presence of a systemic toxin. It has also 


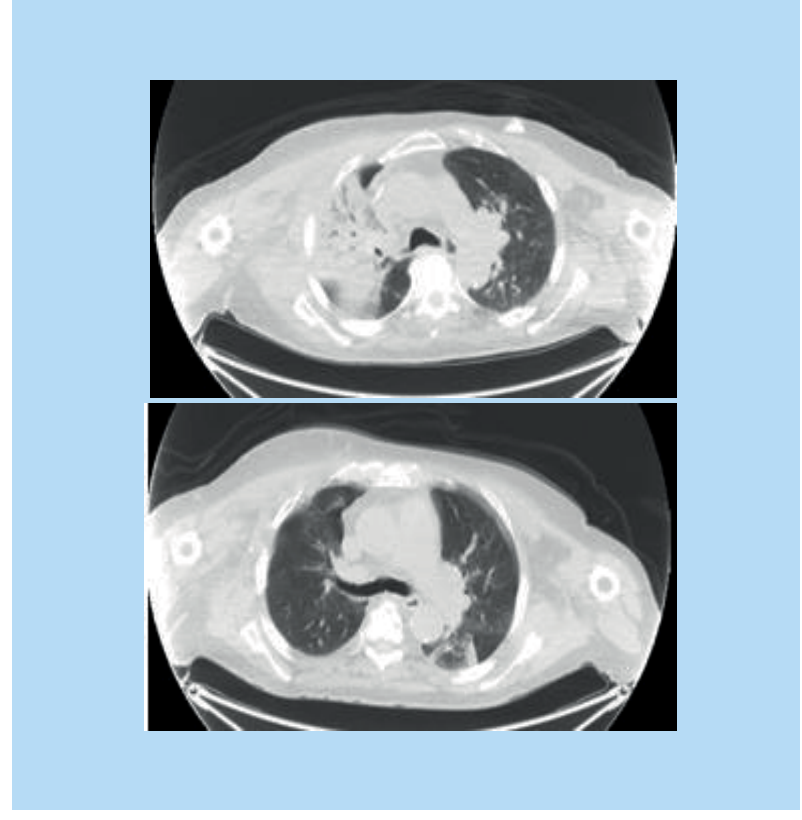

Figure 1. Top image shows initial right upper lobe dense infiltrate suggestive of radiation pneumonitis and bottom image shows resolution of infiltrate 3 months after initiation of prednisone.

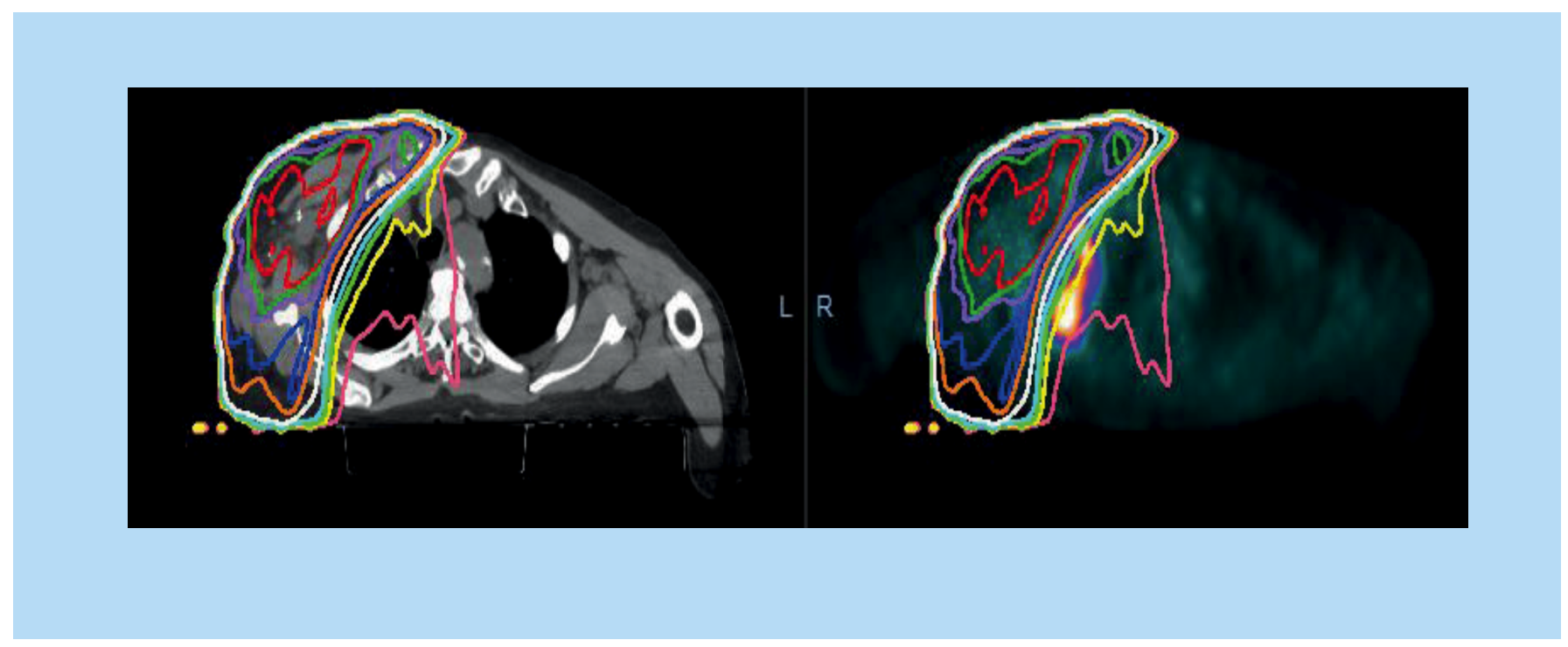

Figure 2. Prior right lateral chest wall mass radiation dosimetry (right) and radiation field overlying the PET/CT scan visualizing the radiation recall pneumonitis (left).

been postulated that radiation therapy may induce hypersensitivity to certain cytotoxic agents. Last, the pharmacodynamics of the systemic agent may be altered by radiation, resulting in elevated concentration of these agents in previously irradiated sites. It is interesting to note that almost all systemic agents that have been reported to induce RRP have also been shown to result in pulmonary toxicity in the form of interstitial pneumonitis and pulmonary fibrosis independent of radiation. Hence, one might theorize that synergistic damage to type II pneumocytes from specific systemic agents and radiation may lead to RRP [1].

\section{Systemic agents associated with RRP}

RRP has been described in the literature in association with several classical chemotherapeutic agents such as paclitaxel [2] and gemcitabine [3]. With the evolution of cancer care using biological and immunological therapies, which are themselves associated with pulmonary toxicities, there seems to be a renewed interest in RRP as an entity. There are recent reports of RRP in patients treated with small-molecule tyrosine kinase inhibitors such as 
Figure 3. Chronic inflammation and Type 2 pneumocyte hyperplasia as seen on endobronchial biopsy.

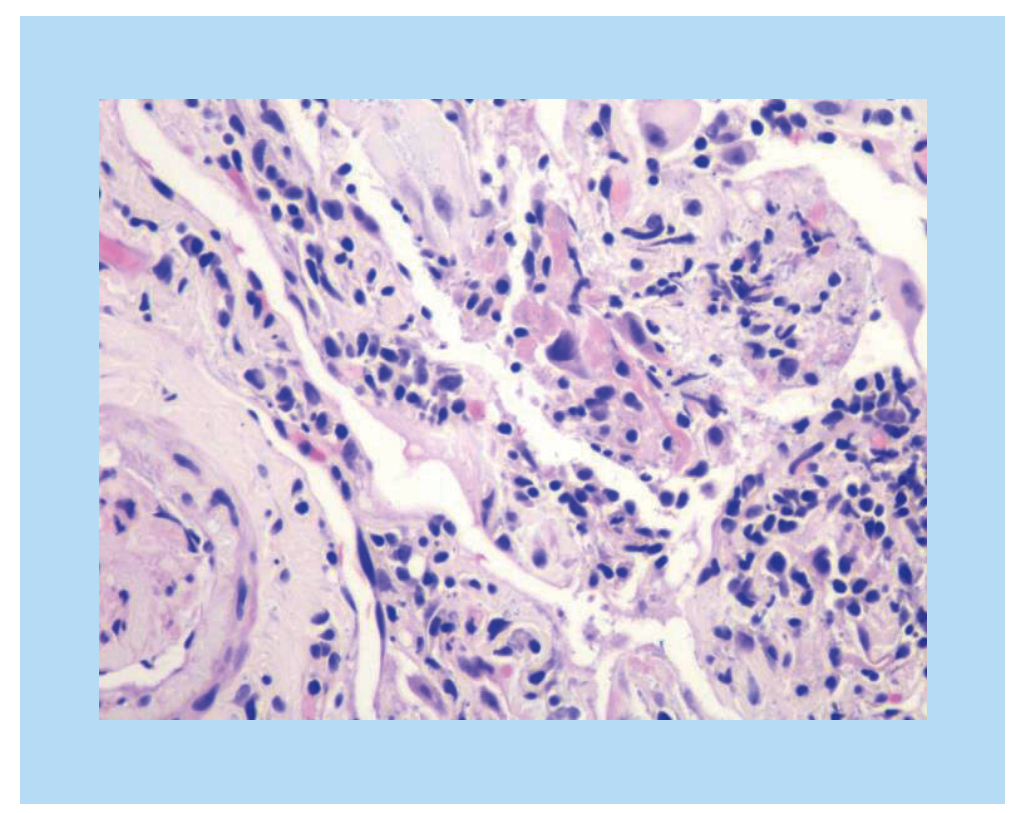

the targeted agents everolimus and sunitinib [4,5]. Recently, a small number of cases of RRP induced by checkpoint inhibitor therapy were reported in the literature [6]. In this manuscript, we report a case of PD-1 inhibitor-associated RRP and present a focused review on the topic.

\section{Chemotherapeutic agents}

RRP has been described in association with several classical chemotherapeutic agents. Azria et al. performed a Medline and Cancerlit search for radiation recall in 2005 and reported taxanes and anthracyclines to be responsible for 20 and $30 \%$ of radiation recall reactions, respectively [7]. Subsequently, Ding et al. published the largest case series of RRP, involving 12 patients [8]. They reported that 8 of the 12 patients in their series had been exposed to taxanes. Other chemotherapeutic agents implicated in RRP include ifosfamide, etoposide, pemetrexed and platinum [9-11].

\section{Biological and other agents}

Several small-molecule kinase inhibitors, particularly those inhibiting the MAPK and mTOR pathways have been associated with pulmonary toxicity. Some of the agents implicated in causing RRP include erlotinib, gefitinib, afatinib, sunitinib and everolimus [12]. These same agents are also linked to RRP in recently published case reports. In 2016, Chiang et al. reported a $4.4 \%$ incidence of RRP in patients with history of prior thoracic receiving EGF tyrosine kinase inhibitor therapy [13]. There are reports of radiation recall with hormonal agents such as tamoxifen as well, though more commonly involving as compared with the lungs [14]. Even nononcological agents such as anti-tuberculosis drugs, statins, antibiotics and antifungal drugs have been reported to cause RRP [15].

\section{A focus on checkpoint inhibitor therapy}

Antibody blockade of the programmed death (PD-1) molecule or its ligand (PD-L1) have revolutionized treatment for various types of cancers including lung cancer, head and neck cancer, melanoma, renal cell carcinoma and lymphoma. By engaging with PD-L1 expressed on tumor cells, these molecules inhibit the immunosuppression rendered by interaction of PD-L1 with PD-1 on T cells, and restore anti-tumor T cell responses [16]. While these agents have shown anticancer activity in a plethora of cancers, they also frequently cause immune-related adverse events. It has been suggested that the tumor neoantigens and normal tissue antigens cross-react and therefore with the introduction of checkpoint inhibitors normal tissue in the GI tract, liver, skin and endocrine organs can be damaged along with the tumor cells [17]. One of the well-documented and potentially fatal immune-related adverse events associated with checkpoint inhibitors is pulmonary toxicity or pneumonitis, with an estimated incidence up to $10 \%$ (any grade) of which $1-2 \%$ are Grade 4 or 5 events [18,19]. Higher rates are seen with combination therapy compared with monotherapy. A recent meta-analysis involving 12 Phase II and III clinical trials (a total of 
6240 patients) evaluated the relationship between risk of pneumonitis and pneumonitis-related death and PD-1 inhibitor treatment in patients with cancer [20]. This study showed a modest increase in relative risk of developing pulmonary complications in patients receiving checkpoint inhibitors compared with patients on chemotherapy (RR $2.65 ; \mathrm{p}=0.06$ ). The incidence of all-grade pneumonitis (Grades $1-4$ ) ranged from 1.3 to $5.8 \%$ whereas high-grade (grades 3-4) pneumonitis ranged from 0 to $2.6 \%$. The incidence of pneumonitis-related death was extremely low $(0-0.6 \%)$.

In clinical practice, we see many patients, particularly those with lung cancer, receiving checkpoint inhibitor therapy who have been previously exposed to thoracic radiation. With the PACIFIC trial, which compared a PD-L1 inhibitor, durvalumab, to observation after chemoradiation in Stage III lung cancer patients, showing significantly improved progression-free and overall survival outcomes [21], we expect that this population will increase tremendously in the near future. Additionally, there are many patients on checkpoint inhibitor therapy who are treated with palliative-intent chest radiation. Hence, it is vital to understand the synergy between these two modalities both in terms of efficacy and toxicity.

Radiation by itself has immunomodulatory effects. It can induce tumor cell death and result in release of damage-associated molecular pattern markers that in turn make tumor antigens more accessible for presentation to cytotoxic $\mathrm{T}$ cells by antigen-presenting cells [6]. Radiation can cause alterations in the tumor cell and tumor milieu in a manner that enhances the sensitivity of tumor cells to immune-mediated cell death. Interestingly, immune-mediated regression of not only the irradiated tumor, but also of distant tumors outside the radiation field (abscopal effect) have been extensively reported [22,23]. The strategy of combining ionizing radiation with checkpoint inhibitor therapy is based on the premise that radiation causes direct cell death releasing tumor antigens and initiates an immune response that is further augmented by checkpoint inhibitor treatment [5].

While there is a strong rationale and background data to support combining immunotherapy and thoracic radiation, there is also significant concern for overlapping pulmonary toxicity. A subgroup analysis from the KEYNOTE-001 trial noted that patients with locally advanced lung cancer who had previously received thoracic radiation had prolonged progression-free and overall survival with pembrolizumab compared with those who had not [24]. At the same time, pooled data from all pembrolizumab studies indicated that pneumonitis was more frequent in patients with a history of prior thoracic radiation compared with those without that exposure (6 vs $2.6 \%)$. It should be noted that the authors of this paper do not specify whether the lung injury was limited to the field of prior irradiation or generalized [25]. In general, cases of pneumonitis associated with immune checkpoint inhibitors have a generalized multilobar distribution. However, the pattern of lung damage with these agents in patients previously exposed to thoracic radiation is not well characterized.

A handful of cases reported in the current literature have described the phenomenon of RRP in patients who received immunotherapy with PD-1 or cytotoxic T cell lymphocyte-associated antigen-4 (CTLA-4) antibodies after prior exposure to thoracic radiation $[26,27]$. Most of these patients had received radiation for a long time, in some cases several years prior to treatment with checkpoint inhibitor therapy. Even though the presenting symptoms varied widely from completely asymptomatic to severely symptomatic with cough, dyspnea and fever, all these patients demonstrated radiographic findings of ground-glass opacities, diffuse haziness, infiltrates or consolidation limited to the previously irradiated fields [28]. The radiological findings are similar to RRP seen with other agents. Histopathological evaluations of these lesions show inflammatory changes such as congested mucosa with leukocytic infiltration, exudative alveolitis, presence of giant cells, hyperplastic type 2 pneumocytes and fibrosis.

In our practice, an 82-year-old gentleman with lung adenocarcinoma developed an asymptomatic fluorodeoxyglucose (FDG)-avid right upper infiltrate in October 2017 after receiving pembrolizumab monotherapy starting in December 2016. His history is notable for prior external beam radiotherapy in August 2016 for a right chest wall mass. He was initially treated with antibiotics with no improvement in radiological findings. While the right upper lobe infiltrate was in the previously irradiated field, most of the areas had received low-dose radiation ranging from 5 to 20 Gy (Figures $1 \& 2$ ). The patient underwent bronchoscopy with endobronchial biopsy of the lesion and pathology showed chronic inflammation and type 2 pneumocyte hyperplasia (Figure 3). Prednisone $1 \mathrm{mg} / \mathrm{kg}$ was initiated followed by a prolonged taper with repeat imaging 3 months later showing resolution of the infiltrate.

\section{Conclusion}

In summary, RRP is a potential risk in patients with prior history of thoracic radiation receiving various systemic agents. This is particularly of concern with checkpoint inhibitor therapies, which are by themselves associated with immune-mediated pneumonitis. Since RRP may mimic cancer progression or pneumonia, there may be 
significant delay in diagnosis. While there are clear guidelines for immunotherapy-induced pneumonitis, there are none for RRP. Risk for RRP should be borne in mind while starting immunotherapy in patients with history of thoracic radiation and a biopsy should be considered when radiological findings are confined to previously irradiated field(s). As demonstrated by our case, RRP is steroid-sensitive and timely initiation of steroids may result in complete resolution of symptoms.

\title{
Future perspective
}

Checkpoint inhibitors are a relatively new therapy in the toolbox for oncologists. There is a plethora of ongoing studies looking into the role of checkpoint inhibitors across several different malignancies. With all of these data that are being gathered, there will also be data evolving as to the potential adverse events associated with these medications. Early data have documented some level of risk in terms of RRP, although it will be important to follow these patients over a longer period of time to see if these rates increase. Accurately quantifying the morbidity and mortality associated with RRP may impact how patients are treated in the future, specifically as to where and when steroids are appropriate. Toxicity assessment in future clinical trials should classify RRP as a separate entity and guidelines should be specifically created for this phenomenon.

\section{Summary points}

- Radiation recall pneumonitis (RRP) is a diagnosis that has been documented with several systemic agents used in the treatment of cancer and it is now starting to be documented with checkpoint inhibitors.

- Both radiation therapy and immune checkpoint inhibitors on their own have immune-mediated adverse effects and therefore the combination of these therapies can lead to synergistic consequences, including pneumonitis.

- There are no specific guidelines for diagnosis and treatment of RRP with treatment extrapolated from immune-mediated pneumonitis secondary to immunotherapy. Moreover, RRP can mimic other lung conditions, which may delay diagnosis. There is a need for trials to specifically evaluate for RRP as an adverse event, which can therefore be utilized to create those guidelines.

\begin{abstract}
Author contributions
K McGovern contributed to the design and acquisition of data as well as revising the manuscript with final approval of the published version. K McGovern agrees to be accountable for all aspects of the work presented in the manuscript. M Ghaly contributed to the acquisition and interpretation of the data as well as played a role in drafting the document. M Ghaly had final approval of the version to be published and agrees to be accountable for all aspects of the work. M Esposito substantially contributed to the acquisition, analysis and interpretation of the data presented in the manuscript. Moreover he revised the document as well as had final approval of the most updated version. Last, he agrees to be accountable for all aspects of the work. K Barnaby played a substantial role in the design and analysis of the data as well as revised the manuscript for important intellectual content. She had final approval of the to-be published version and agrees to be accountable in terms of any issues related to accuracy or integrity. $\mathrm{N}$ Seetharamu has significantly contributed to the conception, design, acquisition and analysis of the date. Additionally, she revised the document in a critical manner, had final approval and agrees to be accountable for all aspects of the work in terms of accuracy and integrity.
\end{abstract}

Financial \& competing interests disclosure

The authors have no relevant affiliations or financialinvolvement with any organization or entity with a financial interest in or financial conflict with the subject matter or materials discussed in the manuscript. This includes employment, consultancies, honoraria, stock ownership or options, expert testimony, grants or patents received or pending, or royalties.

No writing assistance was utilized in the production of this manuscript.

Ethical conduct of research

The authors state that they have obtained appropriate institutional approval and have also followed the principles outlined in the Declaration of Helsinki for research involving humans.

Open access

This work is licensed under the Creative Commons Attribution 4.0 License. To view a copy of this license, visit http://creativecomm ons.org/licenses/by/4.0/ 


\section{References}

Papers of special note have been highlighted as: $\bullet$ of interest; $\bullet \bullet$ of considerable interest

1. Rovirosa A, Valduvieco I. Radiation pneumonitis. Clin. Pulm. Med. 17, 218-222 (2010).

2. Schweitzer VG, Juillard GJ, Bajada CL, Parker RG. Radiation recall dermatitis and pneumonitis in a patient treated with paclitaxel. Cancer 76, 1069-1072 (1995).

3. Jeter MD, Janne PA, Brooks S et al. Gemcitabine-induced radiation recall. Int. J. Radiat. Oncol. Biol. Phys. 53, 394-400 (2002).

4. Clark D, Gauchan D, Ramaekers R, Norvell M, Copur MS. Radiation recall pneumonitis during systemic treatment with everolimus. Oncol. Res. 22(5-6), 321-324 (2014).

5. Yuasa T, Kitsukawa S, Sukegawa G et al. Early onset recall pneumonitis during targeted therapy with sunitinib. BMC Cancer 3, 3 (2013).

6. Lu CS, Liu JH. Pneumonitis in cancer patients receiving anti-PD-1 and radiotherapies: three case reports. Medicine (Baltimore) 96(1), e5747 (2017).

7. Azria D, Magné N, Zouhair A et al. Radiation recall: a well recognized but neglected phenomenon. Cancer Treat. Rev. 31, 550-570 (2005).

8. Ding X, Ji W, Li J, Zhang X, Wang L. Radiation recall pneumonitis induced by chemotherapy after thoracic radiotherapy for lung cancer. Radiat. Oncol. 6, 24 (2011).

9. Murata H, Koyama K, Takezawa Y et al. Radiation recall pneumonitis induced by ifosphamide for malignant soft tissue tumor arising from the chest wall: a case report. Mol. Clin Oncol. 8(4), 592-594 (2018).

10. Kataoka K, Kawamura M, Nishiyama Y et al. A case with delayed-onset radiation pneumonitis suspected to be induced by oral etoposide. Nihon Igaku Hoshasen Gakkai Zasshi 52(5), 641-645 (1992).

11. Hochstrasser A, Benz G, Joerger M et al. Interstitial pneumonitis after treatment with pemetrexed: a rare event? Chemotherapy 58(1), 84-88 (2012).

12. Arakawa $\mathrm{H}$ Johkoh $\mathrm{T}$ Sakai $\mathrm{F}$ et al. Exacerbation of radiation fibrosis with erlotinib: another pattern of radiation recall phenomenon. Jpn J. Radiol. 29(8), 587-589 (2011).

13. Chiang CL, Chen YW, Wu MH, Huang HC, Tsai CM, Chiu CH. Radiation recall pneumonitis induced by epidermal growth factor receptor inhibitor in patients with advanced nonsmall-cell lung cancer. J. Chin. Med. Assoc. 79(5), 248-255 (2016).

- This reference reported rates of radiation recall pneumonitis across a specific class of treatment, which helps pave the way for further studies into different therapeutic classes such as checkpoint inhibitors.

14. Kundranda MN, Daw HA. Tamoxifen-induced radiation recall dermatitis. Am. J. Clin. Oncol. 29, 637-638 (2006).

15. Extermann M, Vogt N, Forni M, Dayer P. Radiation recall in a patient with breast cancer treated for tuberculosis. Eur. J. Clin. Pharmacol. 48(1), 77-78 (1995).

16. Sullivan K, Preeshagul I, Budman D, Seetharamu N. Immune checkpoint inhibitors in lung cancer: an update. Future Oncol. 13(11), 955-959 (2017).

17. Michot JM. Immune-related adverse events with immune checkpoint blockade: a comprehensive review. Eur. J. Cancer 54, 139-148 (2016).

- Michot et al. reviewed potential mechanisms by which radiation recall pneumonitis with checkpoint inhibitors could arise.

18. Khunger $\mathrm{M}$, Rakshit $\mathrm{S}$, Pasupuleti $\mathrm{V}$ et al. Incidence of pneumonitis with use of programmed death 1 and programmed death-ligand 1 inhibitors in non-small cell lung cancer: a systematic review and meta-analysis of trials. Chest 152, 271-281 (2017).

19. Naidoo J, Wang X, Woo KM et al. Pneumonitis in patients treated with anti-programmed death-1/programmed death ligand 1 therapy. J. Clin. Oncol. 35, 709-717 (2017).

20. Cui P-F, Ma J-X, Wang F-X, Zhang J, Tao H-T, Hu Y. Pneumonitis and pneumonitis-related death in cancer patients treated with programmed cell death-1 inhibitors: a systematic review and meta-analysis. Ther. Clin. Risk Manag. 13, 1259-1271 (2017).

-• This meta-analysis looked at over ten studies noting an increase in the rates of pneumonitis in those who had received checkpoint inhibitors.

21. Antonia SJ, Villegas A, Daniel D et al. Durvalumab after chemoradiotherapy in stage III non-small-cell lung cancer. N. Engl. J. Med. 377, 1919-1929 (2017).

22. Formenti SC, Demaria S. Systemic effects of local radiotherapy. Lancet Oncol. 10(7), 718-726 (2009).

23. Tang C, Wang X, Soh H et al. Combining radiation and immunotherapy: a new systemic therapy for solid tumors? Cancer Immunol. Res. 2(9), 831-838 (2014).

24. Shaverdian N, Lisberg AE, Bornazyan K et al. Previous radiotherapy and the clinical activity and toxicity of pembrolizumab in the treatment of non-small-cell lung cancer: a secondary analysis of the KEYNOTE-001 Phase I trial. Lancet Oncol. 18, 895-903 (2017).

-. This article is of importance as it compared rates between those who received radiation and those who did not in an early trial involving pembrolizumab. 
25. Sul J, Blumenthal GM, Jiang X, He K, Keegan P, Pazdur R. FDA approval summary: pembrolizumab for the treatment of patients with metastatic non-small-cell lung cancer whose tumors express programmed death-ligand 1. Oncologist 21, 643-650 (2016).

26. Thore P, Godbert B, Petit I, Chaouat A. Radiation recall pneumonitis in a patient treated by nivolumab for non-small-cell lung cancer, no relapse with rechallenge. J. Med. Oncol. 1(3), 12 (2018).

27. Shibaki R, Akamatsu H, Fujimoto M, Koh Y, Yamamoto N. Nivolumab induced radiation recall pneumonitis after two years of radiotherapy. Ann. Oncol. 28(6), 1404-1405 (2017).

28. Choi YW, Munden RF, Erasmus JJ et al. Effects of radiation therapy on the lung: radiologic appearances and differential diagnosis. Radiographics 24, 985-998 (2004).

- The authors present on the radiologic features of this phenomenon which may be nonspecific or mimic other conditions, making it important to consider this condition in the right clinical context. 
